\title{
FLAMINGO - Fulfilling enhanced Location Accuracy in the Mass-market through Initial GalileO services
}

\author{
William Roberts, Joshua Critchley-Marrows, Marco Fortunato, Maria Ivanovici NSL, UK; \\ Karel Callewaert, Thiago Tavares, VVA Brussels, Belgium; \\ Laurent Arzel, Axelle Pomies, Telespazio France SAS
}

\section{BIOGRAPHIES}

William Roberts is the Operations Manager, Joshua Critchley-Marrows a GNSS Navigation Engineer, Marco Fortunato a Graduate Engineer and Maria Ivanovici a Project Manager at NSL. Karel Callewaert is Senior Consultant and Thiago Tavares Principal Consultant at VVA. Laurent Arzel is GNSS Project Leader and Axelle Pomies Head of Satnav Downstream Department at Telespazio France.

\begin{abstract}
This paper discusses FLAMINGO, an initiative that will provide a high accuracy positioning service to be used by mass market applications. The status and future for the initiative are discussed, the required accuracies and other location parameters are described, and the target applications are identified. Finally, the currently achieved accuracies from today's Smartphones are assessed and presented.

FLAMINGO (Fulfilling enhanced Location Accuracy in the Mass-market through Initial GalileO services), part funded through the European GNSS Agency, is a collaborative venture comprising NSL (as lead organization), Telespazio France, University of Nottingham, Rokubun, Thales Alenia Space France, VVA, BQ, ECLEXYS and Blue Dot Solutions. The initiative is developing the infrastructure, solutions and services to enable the use of accurate and precise GNSS within the mass-market, thereby operating predominantly in an urban environment. Whilst mass-market receivers are yet to achieve accuracies below one metre for standard positioning, the introduction of Android raw GNSS measurements and the Broadcom dual frequency chipset (BCM47755), has presented the devices such an opportunity.

FLAMINGO will enable and demonstrate the future of high accuracy positioning and navigation information on mass-market devices such as smartphones and Internet of Things (IoT) devices by producing a service delivering accuracies of 50cm (at $95 \%$ ) and better, employing multi-constellation, PPP and RTK mechanisms, power consumption optimisation techniques. Whereas the Galileo High Accuracy Service targets $10 \mathrm{~cm}$ precision within professional markets, FLAMINGO targets $30-50 \mathrm{~cm}$ precision in the mass-market consumer markets. By targeting accuracies of a few decimetres, a range of improved and new applications in diverse market sectors are introduced. These sectors include, but are not limited to, mapping and GIS, autonomous vehicles, AR environments, mobilelocation based gaming and people tracking.

To obtain such high accuracies with mass market devices, FLAMINGO must overcome several challenges which are technical, operational and environmental. This includes the hardware capabilities of most mass-market devices, where components such as antennas and processors are prioritised for other purposes. We demonstrate that, despite these challenges, FLAMINGO has the potential to meet the accuracy required. Tests with the current Smartphones that provide access to multi-constellation raw measurements (the dual frequency Xiaomi Mi 8 and single frequency Samsung S8 and Huawei P10) demonstrate significant improvements to the PVT solution when processing using both RTK and PPP techniques.
\end{abstract}

\section{INTRODUCTION}

Our FLAMINGO initiative targets the mass-market and therefore will produce products and services for consumption by very large numbers of people and items. Mass market devices include Smartphones, IoT things and wearables, many of which already include 
some form of GNSS receiver. These are widely produced, low-cost and common receivers utilising low-cost antennas which are often shared with other RF components. Until recently they were only capable of lower accuracy positioning, in the region of 5-10m at best, although other features of the devices have already been utilized for higher accuracy positions within professional markets. This includes maximizing the convenience of the Smartphone as a data collection device, linked to an external GNSS receiver/antenna via Bluetooth or cable (see the [5] as an example). The processor power now available within the Smartphone is also being exploited by Trimble's Catalyst $1-2 \mathrm{~cm}$ solution that is a software defined radio GNSS receiver residing on the handset paired with an external antenna and digitizing RF front end [4].

Several recent developments have created the opportunity for high accuracy positioning directly in the Smartphone. New GNSS capabilities include new constellations and augmentations of GNSS satellites with open signals that are nearing their final stages of deployment, such as the European Galileo, Chinese Beidou, and Japanese QZSS. Also, new signals are being transmitted from existing GNSS constellations, such as the L5 frequency from GPS satellites. In 2016, Google announced the availability of raw GNSS measurements through an API on their Android 7 operating system [1] which, when implemented by the chipset, modem and handset manufactures, allow the extraction of pseudorange, carrier phase, Doppler measurements which are the core components of high accuracy GNSS positioning techniques. In September 2017, Broadcom released the BCM47755 that provides multiconstellation (GPS, Galileo, GLONASS, Beidou and QZSS) GNSS tracking capabilities on L1 along with GPS L5, Galileo E5a and QZSS L5. Additionally, in January 2018, the GSA published the Raw Measurements White Paper that provided guidance on how to access and combine the Google raw measurements and to subsequently use the information for high accuracy positioning.

Smartphones soon became available that supported these raw measurements and specifically, the Huawei P10 and Samsung S8 providing single frequency carrier phase and pseudorange measurements for GPS, GLONASS, GALILEO, BeiDou, and QZSS. In May 2018 a further device, the Xiaomi Mi 8, become the first Smartphone to use the BCM47755 and support dual frequency observations though the inclusion of L5/E5a for GPS, Galileo and QZSS satellites. The devices and initiatives are challenging the ways in which we can obtain high accuracy positioning which in turn creates new applications and uses.

It is important to note that high accuracy positioning for mass market devices is at its infancy. The aforementioned technology triggers have led to academic R\&D with experimental solutions that demonstrate the potential. To become mass market, handset manufacturers need to integrate the new GNSS chipsets with raw measurements into their devices (first exhausting legacy GNSS chipsets) and subsequently there will be a period of device churn before they become commonplace in the market. Service providers need to establish infrastructure, develop positioning services and solutions, and developers need to integrate this into their applications.

The purpose of FLAMINGO is to develop these first-generation services and solutions and to demonstrate to key stakeholders with the intention that they become early adopters. Demonstrations to stakeholders will be in the form of long duration showcase events at three locations across Europe and developer engagement will be through participation in, and theme sponsorship of, technology hackathons aimed at developers of recreational activity, logistics, gaming, professional and lifestyle applications.

\section{APPLICATIONS OF FLAMINGO}

The project partner VVA led a comprehensive analysis of applications that could be relevant to the FLAMINGO system across a broad range of markets and industries. Each application was explored in terms of size of the market and required positioning capabilities including factors such as time-to-first-fix, time-to-first-precise-fix, location accuracy, availability and authenticity. Other aspects were also considered, for instance user demographics and tolerance to outages which are especially important when looking at consumer applications such as gaming and virtual reality.

The analysis led to identifying a target accuracy of $50 \mathrm{~cm}(1 \sigma)$ for FLAMINGO along with a series of applications that should be enabled by FLAMINGO (horizontal accuracy $<50 \mathrm{~cm}$ ) should benefit from FLAMINGO (horizontal accuracy around $1 \mathrm{~m}$ ) and those which have a limited market size (market size below 100,000). The applications are summarized in Figure 1. Statistics are based on a provisional assessment of the potential number of users in Europe and have been collected and collated from [6-12].

The most significant area identified in Figure 1 is Mapping and Geographic Information System (GIS). This application requires accuracies in the lower decimeters and holds an estimated market size of 310 million users in Europe alone. This high statistic is generated by users of the Google Maps app, which is one of the most used apps on the smartphone. Google recently reported 
Google Maps exceeding 1 billion active users. Also, Google Play quotes there are 47,000 free Maps \& Navigation applications alone. Currently, however, mapping and GIS applications in IOT and smartphones is limited to accuracies over a meter and is restricted in terms of availability in the urban canyon. Improving this accuracy will allow users to explore their environment with a greater awareness, such as the end of a sidewalk or the lane of a highway. In GIS, an amateur surveyor may be able to map the size of their garden or driveway. Currently, such capabilities are unavailable in mass-market receivers.

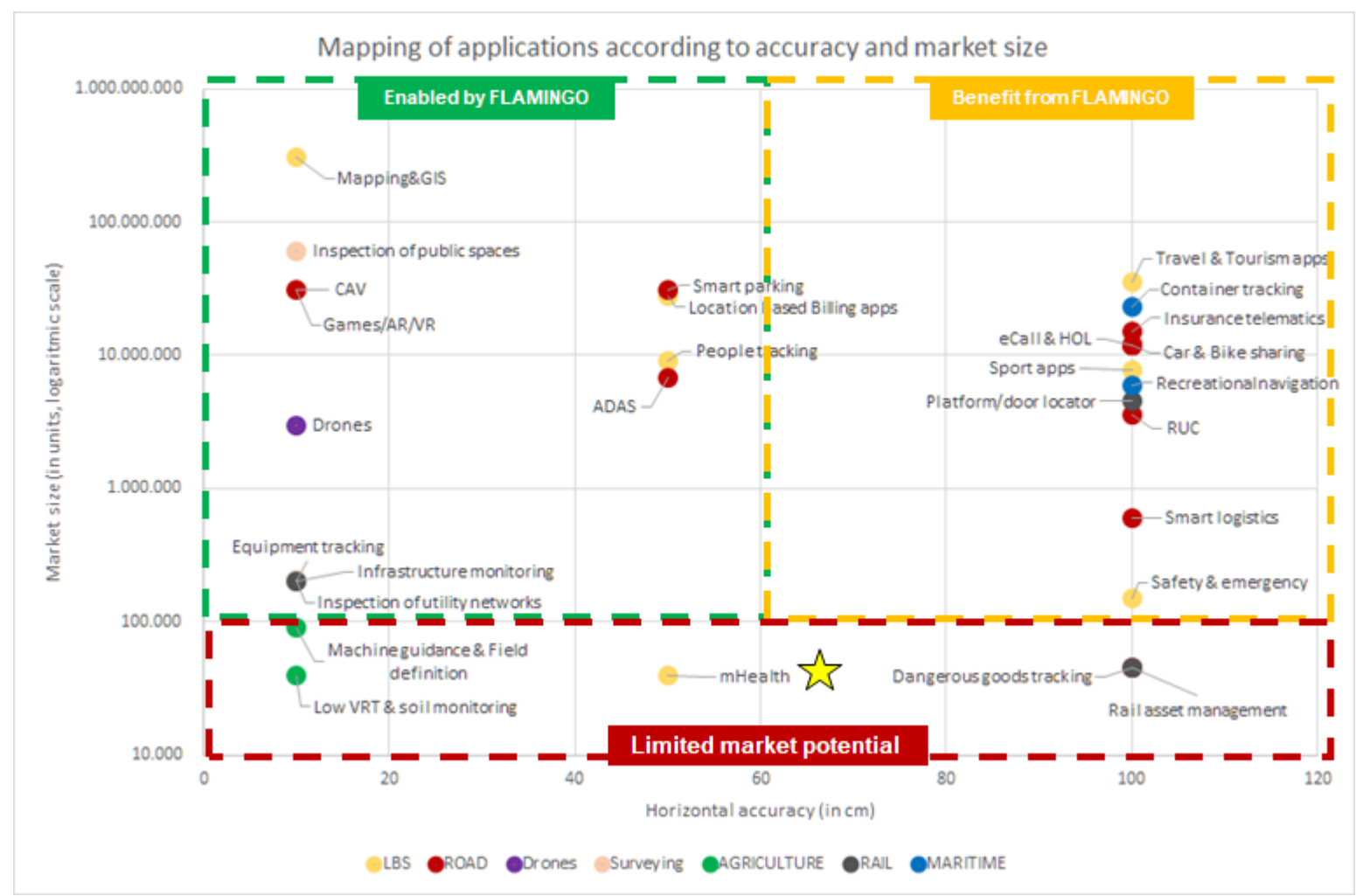

Figure 1: Overview of FLAMINGO target applications, provided in terms of horizontal accuracy and market size.

Mobile Location Based Gaming is another key application. The potential of the gaming market to use location-based services is growing rapidly, largely kickstarted by the release of Pokémon Go in 2016, which saw over 100 million downloads in the first few months. Augmented reality games heavily rely on GNSS, allowing the user to interact with the real world using augmented features, such as a game creature or a virtual shop, placed at certain GNSS coordinates. The market value of video games was estimated in 2017 to be US\$3,710m.

Location based games would require at least $1 \mathrm{~m}$ of accuracy, with a Time-To-First-Fix (TTFF) below 30 seconds. This will be achieved by the FLAMINGO system. If Augmented Reality (AR) is used however, the positioning accuracy must be in the low decimeters, up to $10 \mathrm{~cm}$. Otherwise, the virtual objects which the user will interact will not be visually seamless in the environment. These accuracies refer again to the challenges of FLAMINGO, which will be discussed in the next section.

FLAMINGO may also be targeted for applications concerning public safety, where people tracking is a critical market identified within market analysis. Parents are known to often lose sight of their children, and currently their lacks a reliable, accurate product to keep track of their whereabouts. The accuracies required for this tracking are around 50 to $100 \mathrm{~cm}$ and must operate in congested environments.

People tracking technology can also be applied to the elderly, especially those diagnosed with dementia or other mental illnesses. Care facilities need constant, reliable knowledge of their patients' movements, which FLAMINGO will enable. People tracking apps 
hold approximately 9 million users and is forecasted to grow significantly over the next few years as location services improve and become more reliable.

Some applications have been identified that are enabled yet are slightly out of scope of FLAMINGO at this time. This includes safety critical applications within the autonomous and connected vehicle market and the drone/UAV market.

Identifying the success of FLAMINGO in various markets is an ongoing development. Stakeholder consultation is ongoing to identify potential early-adopters of FLAMINGO in different sectors. Final potential market size will be estimated based on the outcomes of discussions with potential clients.

\section{TECHNOLOGY AND CHALLENGES}

FLAMINGO will provide solutions for high accuracy positioning on Smartphones, IoT and wearables. Not only does this include the positioning engine(s) but the services and infrastructure to provide the necessary data products and the interfaces for developer integration.

The FLAMINGO utilizes many GNSS assets to improve positioning services. These include: multi-constellation, multi-signal GNSS, Precise Point Positioning (PPP), Real Time Kinematic (RTK), hybridized positioning using other non-GNSS sensors, rapid correction products and specially developed reference station infrastructure.

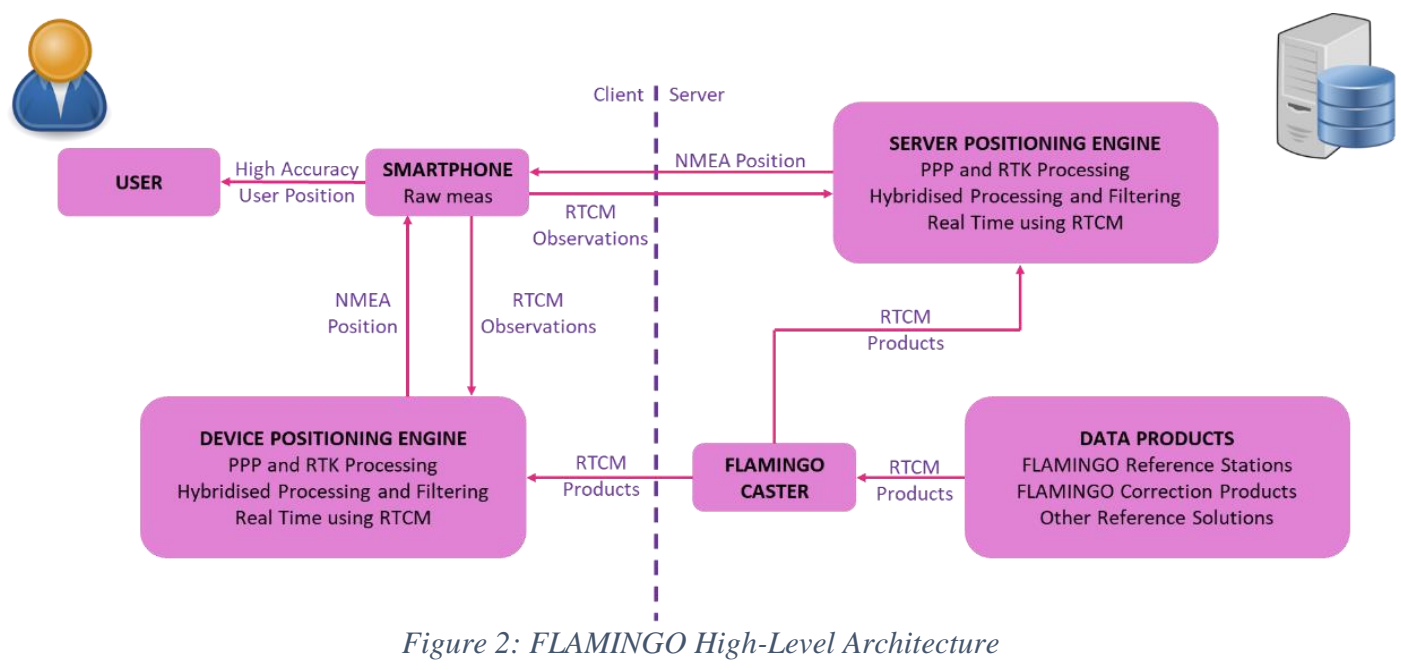

A generalized overview of the architecture is provided in Figure 2 showing both a server and user side to the implementation. Both RTK and PPP reside on both platforms which will permit high accuracy positioning on all devices irrespective of processor capability. Performance of these algorithms is dependent on additional GNSS infrastructure, capable of delivering multi-GNSS orbits and clock information, atmospheric delay models from reference networks and other rapid data products. Data delivery and communications will be predominantly based on RTCM over NTRIP, the common format and protocol used within high accuracy professional positioning. The infrastructure is currently under development.

The technological challenges that FLAMINGO must overcome are considerable. Mass market devices, by definition, are operated mainly in densely populated urban environments. Such environments are not ideal in terms of GNSS signal reception due to blockage, attenuation, and reflection from buildings and other objects (vehicles, people, furniture, etc).

The device itself is also problematic. The most prevalent example of mass market GNSS receivers is the Smartphone which can be considered as an entertainment, communications and photography/video device. Priority of the quality and performance of internal components is based on these key features. Consequently, the GNSS chipset, local oscillator and antenna used are relatively lowgrade. In addition, the chipset is surrounded by other RF components that will induce noise and interference to the GNSS signals. 
The main restriction to high-accuracy is the antenna used inside the smartphone. Smartphone antennas are optimized for various telecommunication interfaces such as $3 \mathrm{G}, \mathrm{WiFi}$ and Bluetooth, and not for GNSS signal tracking. The limitations of this design lead to a reduction in important features which are common for survey-grade antennas, i.e. right-hand polarization, low axial ratio, uniform quasi-hemispherical gain pattern and constant phase-center. This results in a lower sensitivity [13] and reduced capabilities to multipath suppression. This is especially significant in an urban environment, where indirect GNSS signals coming from buildings and other environmental features significantly increase multipath errors and prevent centimeter accuracy [15]. The limitations of the antenna are highlighted by research showing significant performance improvements when an external antenna is utilized [13-14].

For this reason, the multipath-induced phase errors cause more issues in reaching high accuracy than just low signal to noise ratios [14-15]. The result is that low-cost GNSS chipsets and antennas are represented by noisier observations then those obtained from traditional GNSS devices and, consequently, the success-rate of ambiguity fixing decreases [13-16]. Additionally, the low-quality temperature-compensated crystal oscillator used in a mass-market receiver leads to low-frequency phase errors and cycle-slip repairing algorithms are difficult to implement with these errors [15] and by the quality of the shared antenna.

Not only is GNSS a minor portion of the complex smartphone device, which involves hundreds of various internal processes running concurrently, but the user is also severely restricted in control capabilities. The duty cycle is a clear example of this restriction to user input. The duty cycle is an autonomous power-saving mode implemented by Android's internal battery and power optimization software. The consequence of this autonomous process is a jump in carrier-phase, since the process leads the GNSS chipset to switch off the RF section. For some, the duty cycle limited analysis to some initial studies into Android's raw GNSS measurements API [17-18], where the carrier-phase analysis was restricted to only the first few minutes of the dataset.

An additional method in the raw measurement API released with Android N allows the user to disable duty cycling. For earlier versions of the OS and API, duty cycling can be disabled by regularly requesting the navigation message from the chipset. Under such circumstances the GNSS chipset is unable to turn off the RF and thereby duty cycling is disabled.

Despite these challenges studies (in FLAMINGO and elsewhere) have proven that mass-market receiver can achieve high accuracy results. However, they do not, and will not, compete with a survey-grade GNSS receiver.

\section{INITIAL SMARTPHONE PERFORMANCE ANALYSIS}

Data was collected in an anechoic chamber in order to assess the performances of various smartphones removing the impact of environmental effects, as discussed previously. A navXperience $3 \mathrm{G}+\mathrm{C}$ external survey grade antenna is used to transmit the GNSS signal into the anechoic chamber where the signal is amplified. This experimental environment allows us to evaluate the performance of the GNSS chipsets and antennas in a controlled setting. The anechoic chamber effectively disables other RF processes in the Smartphone significantly reducing electronic interferences.

Forty-five minutes of data, measured at a frequency of $1 \mathrm{~Hz}$, is collected from a dual frequency Xiaomi Mi 8, and single frequency Samsung S8 and Huawei P10 using the rinexON app, a dual-frequency observation and navigational RINEX file logger developed at NSL and available on Google Play. rinexON has the advantage over other loggers as it regularly requests the navigation message from the chipset and therefore duty cycling is not present within any of the data.

Specifications of these smartphones are detailed in Table 1. The measurements were collected on the $30^{\text {th }}$ of August 2018 with only one smartphone at time within the anechoic chamber to avoid any interference between different devices. 
Table 1: Specifications of the Xiaomi Mi 8, Samsung S8 and Huawei P10 smartphones

\begin{tabular}{|c|c|c|c|}
\hline Specifications & Xiaomi Mi 8 & Samsung S8 & Huawei P10 \\
\hline OS & Android 8.1 & Android 8.0 & Android 8.0 \\
\hline CPU & $\begin{array}{l}\text { Qualcomm SDM845 } \\
\text { Snapdragon } 845\end{array}$ & Exynos 8895 Octa & HiSilicon Kirin 960 \\
\hline CPU Chipset & $\begin{array}{l}\text { Octa-core }(4 \times 2.8 \mathrm{Ghz} \text { Kyro } \\
385 \text { Gold and } 4 \times 1.8 \mathrm{GHz} \\
\text { Kyro } 385 \text { Silver })\end{array}$ & $\begin{array}{l}\text { Octa-core }(4 \times 2.3 \mathrm{Ghz} \\
\text { Mongoose M2 and } 4 \times 1.7 \\
\text { GHz Cortex-A53) }\end{array}$ & $\begin{array}{l}\text { Octa-core }(4 \times 2.4 \mathrm{Ghz} \text { Cortex- } \\
\text { A73 and } 4 \times 1.7 \mathrm{GHz} \text { Cortex- } \\
\text { A53) }\end{array}$ \\
\hline GNSS Chipset & Broadcom BCM47755 & Broadcom BCM4774 & Broadcom BCM4774 \\
\hline GNSS Signals & $\begin{array}{l}\text { GPS L1, L5, GLONASS L1, } \\
\text { Galileo E1, E5 }\end{array}$ & $\begin{array}{l}\text { GPS L1, GLONASS L1, } \\
\text { Galileo E1 }\end{array}$ & $\begin{array}{l}\text { GPS L1, GLONASS L1, } \\
\text { Galileo E1 }\end{array}$ \\
\hline
\end{tabular}

The performances are evaluated using zero-baseline RTK and PPP. The zero-baseline is achieved through a Septentrio PolaRx5x receiver connected to the same external antenna via a splitter, allowing us to remove all baseline-length dependent errors and to focus on the evaluation of positioning results in ideal conditions. PPP processing is based on rapid predicted orbits and ionospheric corrections provided, respectively, by IGS and JPL. Both analyses are executed considering GPS and Galileo constellations with a cut-off angle equal to 10 degrees.

\section{Comparative RTK Analysis}

To compare like-for-like RTK results from all Smartphones only single frequency L1/E1 data has been considered. Data postprocessing is executed in a real-time scenario considering broadcast ephemeris and clock, broadcast ionospheric corrections and use of the Saastamoinen model to correct hydrostatic tropospheric delay. Only float solutions have been obtained.

The horizontal accuracy is evaluated through statistical analysis of the residuals obtained epoch-by-epoch with respect to the reference coordinates. Different statistical indicators are considered to evaluate both the precision and accuracy.

The results obtained after one minute of convergence in the RTK Kalman filter are displayed on the scatter plot with the origin fixed at the true position. This is presented in Figure 3. The R95 represents the measure of the precision and the displacement from the true position at the origin. This provides an estimate of the accuracy achieved 95\% of the time. The outliers seen in Figure 3 are due to the convergence time required by the Kalman filter. However, even one minute of convergence is not enough to reach the decimeter level. Figure 4 shows that the Xiaomi Mi 8 solution is close to decimeter accuracy for the horizontal components after two minutes. Similar behavior is observed also for the Samsung S8 and the Huawei P10. Vertical accuracy is significant less than the horizontal components of East and North. Figure 5 shows an overview of the precision and accuracy achievable in terms of the mean, standard deviation and RMSE obtained for the three handsets after one minute of convergence time.

Except for the East component, the Xiaomi Mi 8 provides higher precision with respect to the Samsung S8 and the Huawei P10, each containing the older BCM4774 GNSS chipset. The level of accuracy achieved with the Xiaomi Mi8 and the Huawei P10 is comparable but the capabilities of the new BCM47755 is clear in the vertical direction. In Figure 5, the RMSE observed is nearly 24 $\mathrm{cm}$ more accurate. The Samsung S8, on the other hand, performs much worse, especially in the vertical components where the RMSE reaches almost $1.78 \mathrm{~m}$. This difference in overall accuracy is not related to the external environment as test conditions are constant. It must therefore be related to the chipset and an internal hardware-related or software-related issue.

Figure 6 shows the number of GPS and Galileo satellites used to obtain the solution epoch by epoch. The number of satellites used by the Samsung S8 is significantly less when compared to the other two devices. Changes in the number of satellites can lead to a change in the GDOP. This change has a significant impact to the final solutions of the Kalman filter. The average GDOP for this experiment is 2.5 for the Samsung S8 and 1.8 for both the Xiaomi Mi 8 and Huawei P10. This difference highlights a significant challenge to FLAMINGO, as the user has no control over the number of satellites detected. 


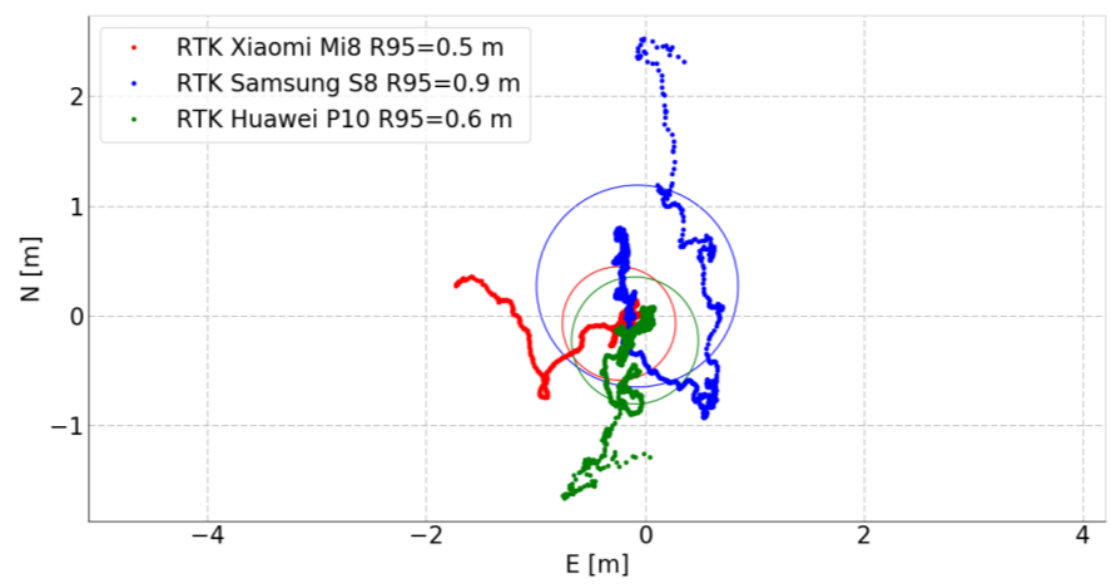

Figure 3: RTK scatter plot for the Xiaomi Mi 8 (red), Samsung S8 (blue) and Huawei P10 (green). The circle represents the R95, i.e. the radius of circle containing the $95 \%$ of the solutions.
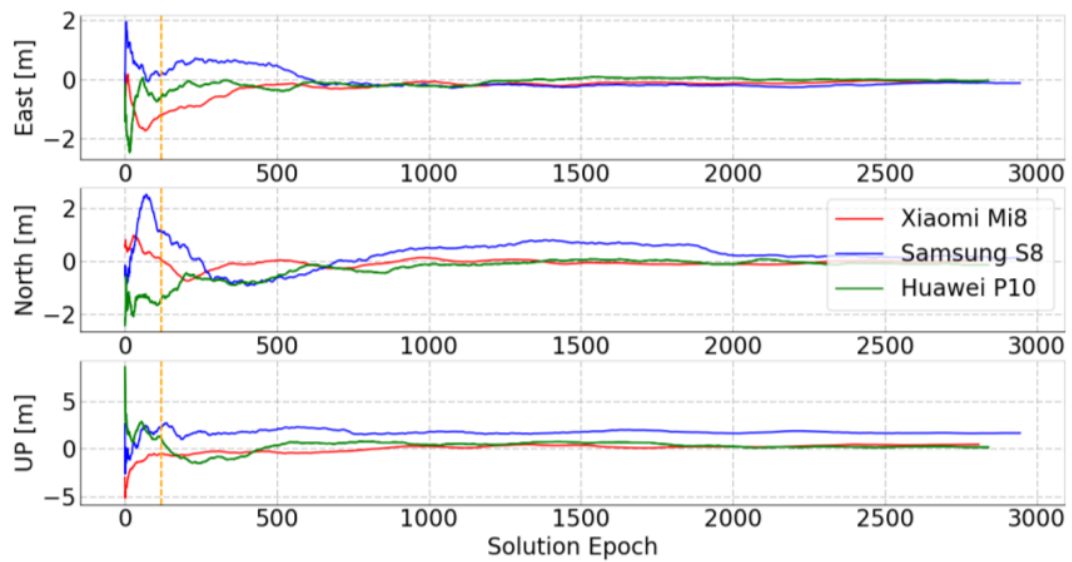

Figure 4: Residuals observed for the for the Xiaomi Mi 8 (red), Samsung S8 (blue) and Huawei P10 (green). The vertical orange line represents the limit of two minutes convergence time necessary to reach decimeter horizontal accuracy. The accuracy for the vertical component is lower, especially for the Samsung $S 8$.

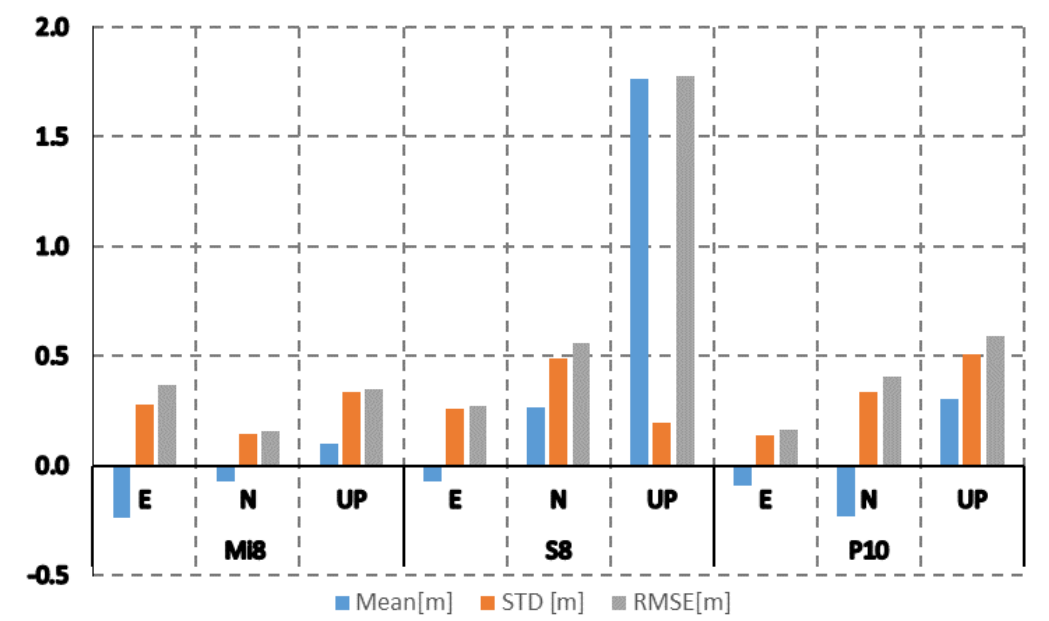

Figure 5: RTK Statistics for the Xiaomi Mi 8, Samsung S8 and Huawei P10. For each device the mean value (blue) is with respect to the true position, the standard deviation is with respect to the mean value (orange) and the final RMSE (grey) is included 


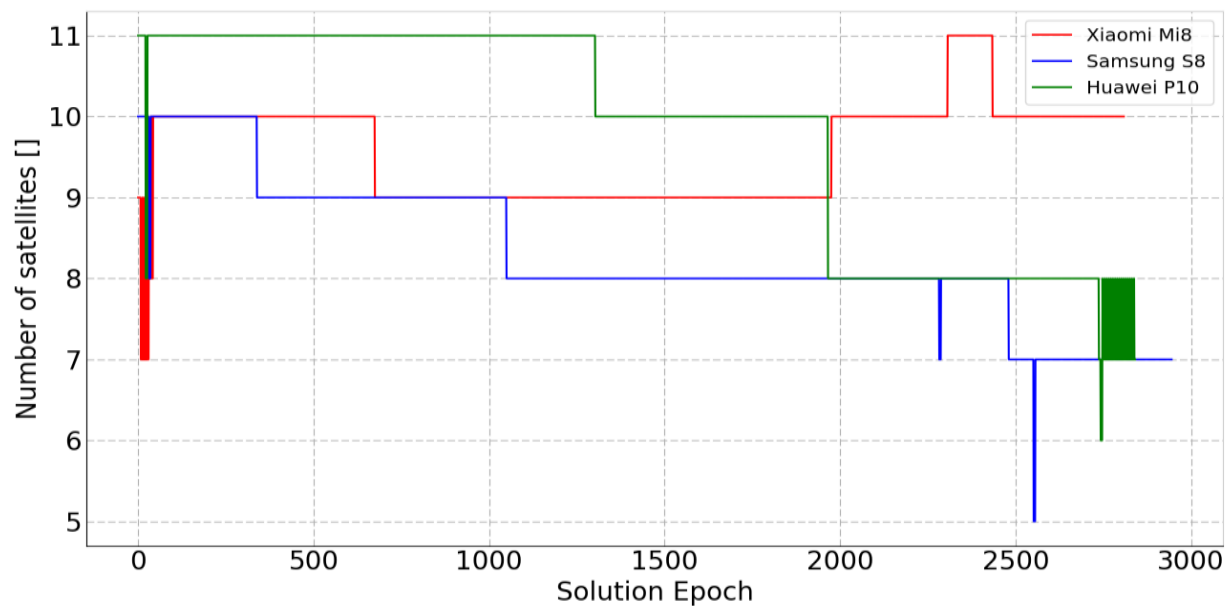

Figure 6: Number of GPS and Galileo satellites used to compute the solutions for the Xiaomi Mi 8 (red), Samsung S8 (blue) and Huawei P10 (green). With relation to the accuracies computed, the number of satellites used by the Samsung S8 is lower, especially after almost 1000 epochs, than the number of satellites used by either the Xiaomi or Huawei handset.

High accuracy positioning through RTK was compared to position that the GNSS chipset reports through the normal geolocation API. Positioning performances of mass-market receivers are also evaluated by comparing results of the RTK algorithm and the internal PVT solutions. Figure 7, Figure 8 and Figure 9 are scatter plots of the RTK and internal PVT solutions for the Xiaomi Mi 8, Samsung S8 and Huawei P10 respectively. The statistics are also presented in Table 2. The R95 is almost 5 times lower in the horizontal coordinate when the position is computed through RTK positioning. Decimeter level of accuracy, which is the target for FLAMINGO, cannot be achieved through the internal PVT solution.

The analysis of Figure 8 highlights also that the internal solution computed from the Samsung S8 is less accurate and precise than those calculated from the Xiaomi Mi 8 and Huawei P10. Considering the controlled environment used for the data collection, this difference can be due only to the varying internal hardware configurations. This can have an impact also on the GNSS raw measurements leading to low accuracy RTK results, as in Figure 3.

Only single frequency observations have been included within the RTK solution and are assumed to be used within the receiver's navigation solution. Figure 7 shows the benefit of the second frequency in the Mi 8 providing cleaner (reduced multipath) L1/E1 benefits. Improved tracking and reduced multipath are key features of the BCM47755. 

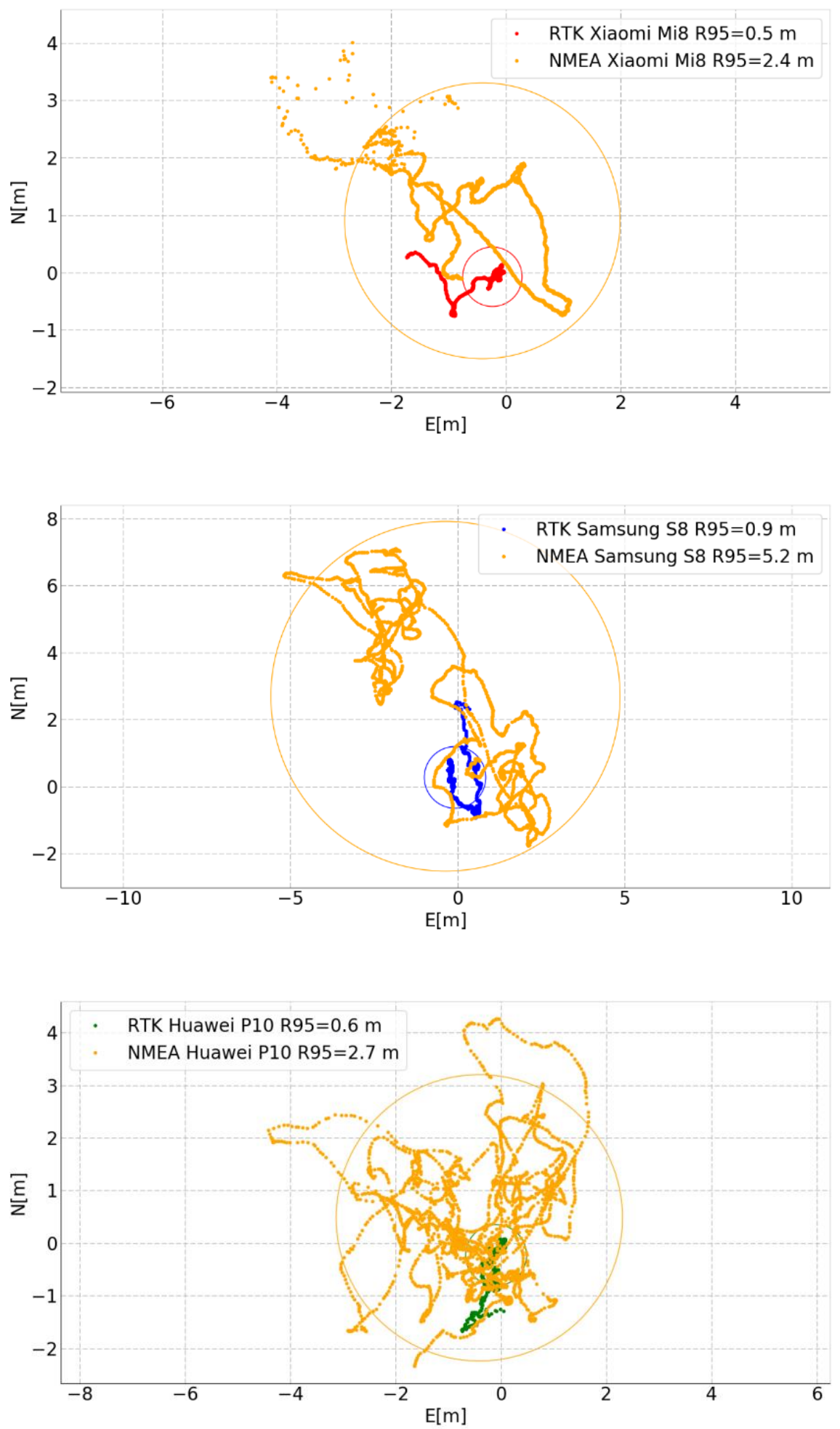

Figure 7: Comparison between the RTK solution (red) and the internal PVT solution (orange) provided by the NMEA messages for the Xiaomi Mi 8.

Figure 8: Comparison between the RTK solution (blue) and the internal PVT solution (orange) provided by the NMEA messages for the Samsung S8.
Figure 9: Comparison between the RTK solution (green) and the internal PVT solution (orange) provided by the NMEA messages for the Huawei P10 
After the analyses of the L1 observations, the features of the new BCM47755 are considered thereby analyzing the benefits brought by dual frequency. The new chipset introduces GPS L5 and Galileo E5a (as well as QZSS L5 that isn't available in the test area). These observations are used in RTK processing using broadcast ionospheric model.

Figure 10 presents a sky plot of the GPS and Galileo satellites tracked by the Xiaomi Mi 8. The satellites are divided into two classes depending on their ability to track only L1/E1c or also L5/E5a. The comparison between the statistical parameters obtained for the residuals of an RTK positioning session with the Xiaomi Mi 8 are presented in Figure 11. These are provided with respect to the reference coordinates of a zero-baseline using either L1 or L1 and L5 observations. Even if the number of satellites providing L5 observations is not high for all epochs considered, in this initial test, an improvement of $33.6 \%, 32.3 \%$ and $43.9 \%$ is observed respectively in the East, North and Up component in terms of the root mean square error. Interestingly, even few satellites with L1 and L5 observations at proper elevation angles can be useful to increase the overall accuracy. Figure 12 illustrates the variation of the residues obtained with the Xiaomi Mi 8 using only L1 observations or both L1/L5 measurements inside the RTK. These results highlight the reduction in convergence time and a more stable final solution by use of L5 observations. Since the Galileo satellites are at low elevations during the first few epochs, these initial observations are removed. However, even with only 3 GPS L5 observations, the advantages coming from the use of this new GNSS chipset are clear. GPS L5, and especially Galileo E5a observations, on the smartphone represent a milestone in the development of high accuracy for GNSS mass-market receivers.

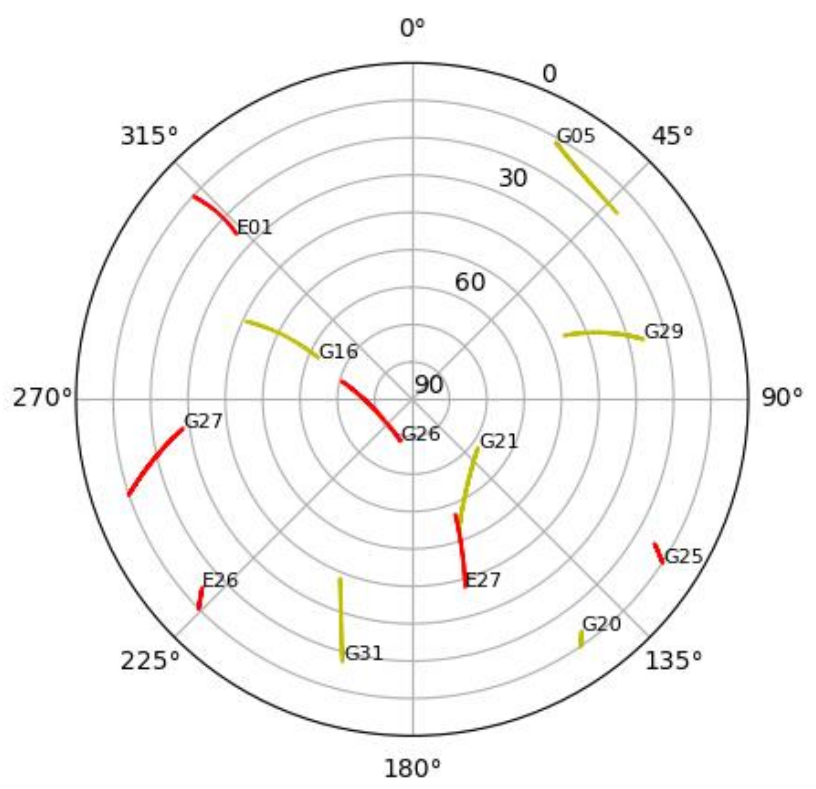

Figure 10: Satellites in view from the Xiaomi Mi 8. The satellites that provide only L1 observations are displayed in yellow. Red is used to represent the satellites providing L1/E1 and L5/E5 observations 


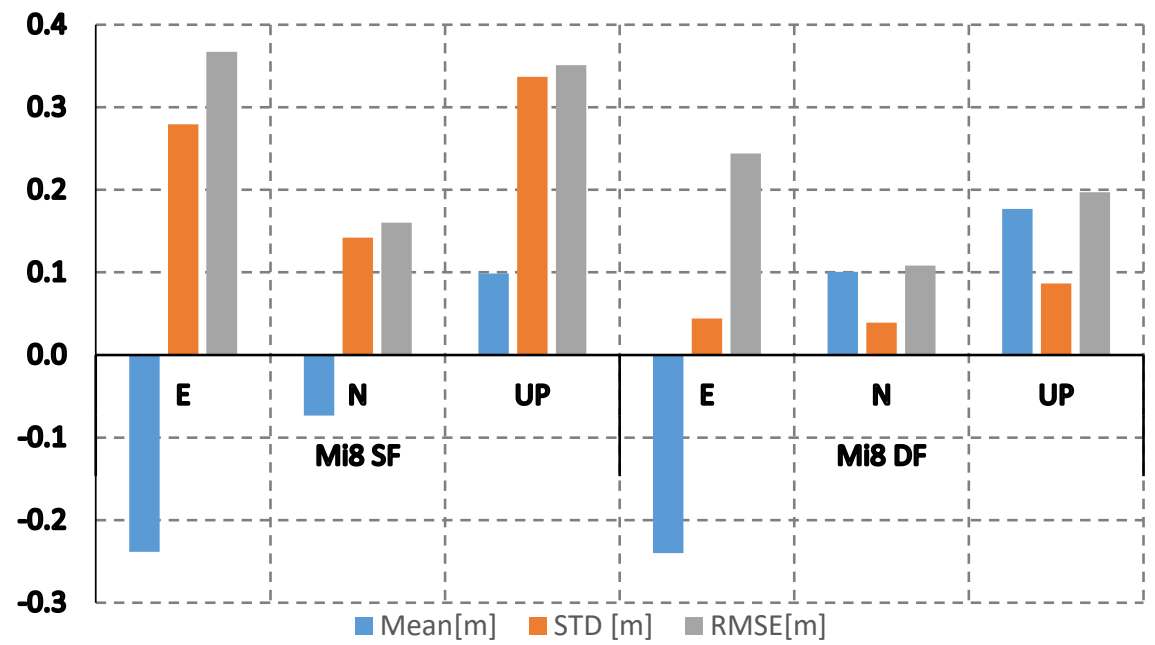

Figure 11: RTK Statistics for the Xiaomi Mi8 results obtained using only L1 observations (Mi 8 SF) and using L1/L5 (Mi 8 DF). Included is the mean value (blue) with respect to the true coordinates, the standard deviation with respect to the mean value (orange) and the final RMSE (grey).

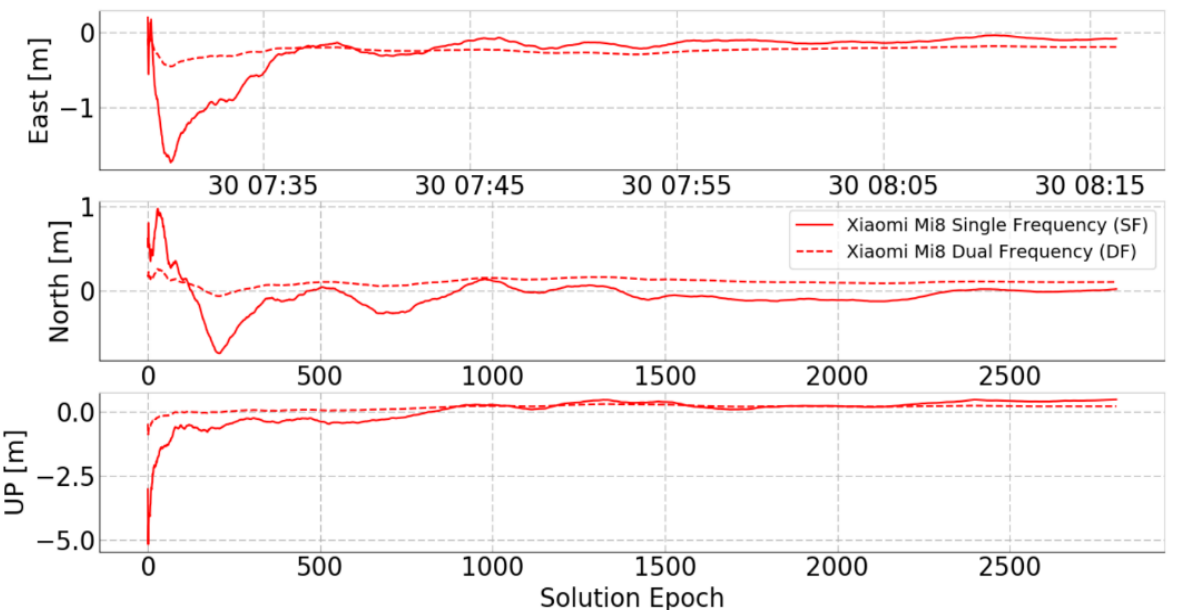

Figure 12: Comparison between the RTK single frequency (bold line) and dual frequency (dotted line) solutions obtained for the Xiaomi Mi8.

\section{Comparative PPP Processing Analysis}

Post-processing analysis using PPP is also considered, employing rapid calculated orbits. The performances of the three different smartphones, specified in Table 1, are compared using the results obtained from a PPP analysis on the L1/E1c frequency. The ionospheric delay is corrected by using the rapid ionospheric correction provided by JPL.

As in the RTK case, the horizontal precision and accuracy is evaluated through the statistical analysis of the residuals obtained epochby-epoch with respect to the reference coordinates. Figure 13 shows the mean, standard deviation and RMSE obtained from the PPP solutions for each device. The results of this analysis are also presented in Table 2. For all the handsets considered in Figure 13, the accuracy obtained from the PPP analysis is lower than those obtained from the RTK analysis. The initial guess of the Kalman filtermeasurement noise and initial coordinates - are the same. So, the differences observed between RTK and PPP may be related to differences in models, where the main errors that have to be considered within the PPP are removed in short baseline RTK positioning, i.e. the ionospheric and tropospheric delays. 
The PPP algorithm, especially without high accurate ionospheric corrections, require a higher convergence time as can be observed in the scatter plot in Figure 14. This is evident by the extensive trend in calculated positions in the plot, as well as by the significantly larger initial displacement from the true. One of the most distinctive features with dual frequency observations is the possibility to include an ionospheric-free linear combination in processing. This allows us to remove the first order ionospheric delay, which is the main impediment to achieving a high accuracy within GNSS positioning. Unfortunately, the number of L5/E5a observations with an elevation higher than 10 degrees is low, so evaluating the improvement in elimination of the ionospheric effect cannot be evaluated with this dataset. If both L1/E1c and L5/E5a observations from low elevation satellites are available, solutions can be obtained by removing the cut-off threshold. This is however unreliable and develops outliers.

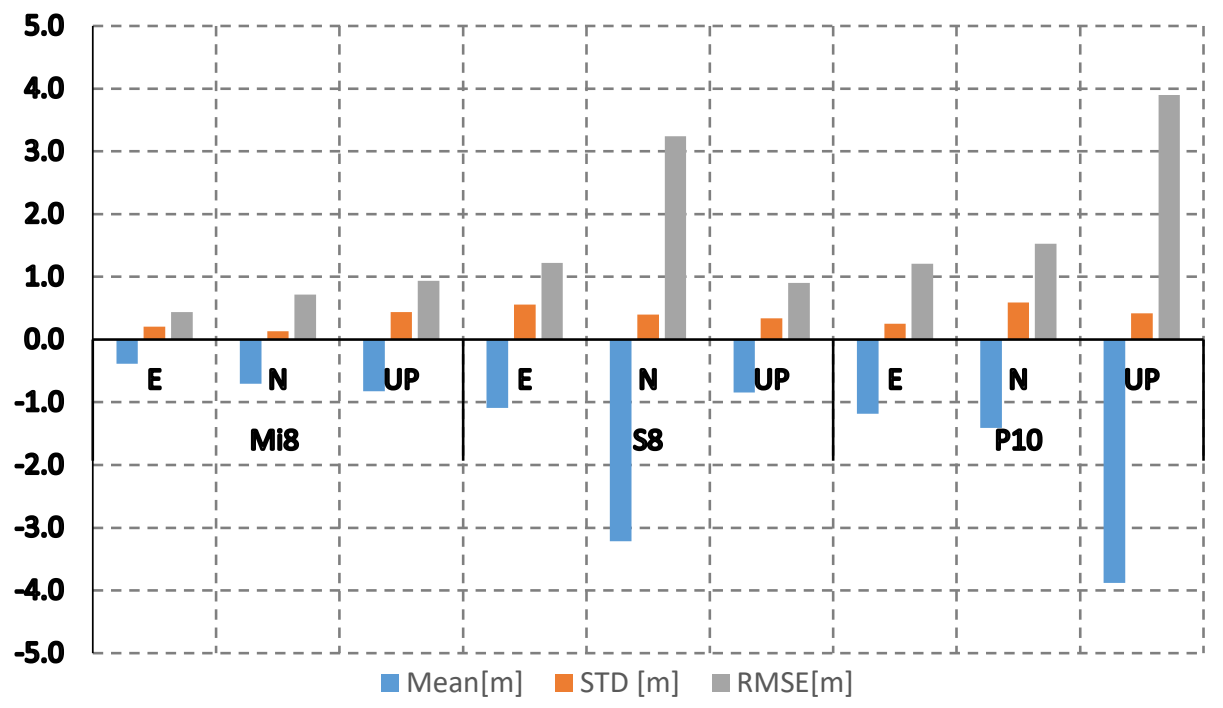

Figure 13: Single frequency PPP statistics for the Xiaomi Mi8, Samsung S8 and Huawei P10. For each device, the mean value (blue) with respect the true coordinates, the standard deviation with respect the mean value (orange) and the final RMSE (grey) are presented

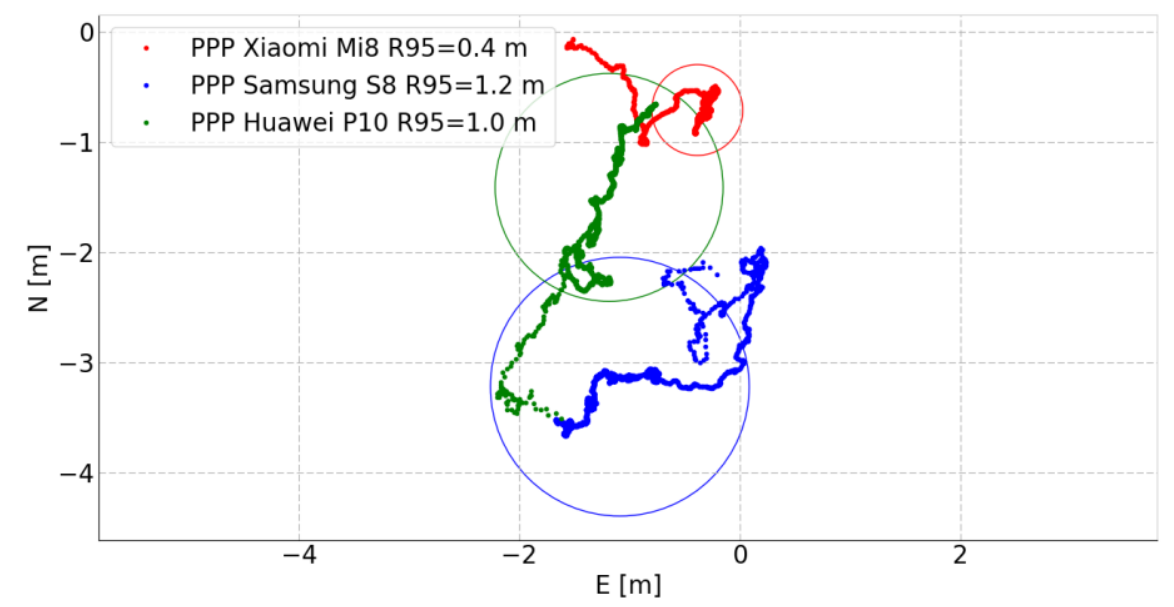

Figure 14: Single Frequency PPP scatter plot for the Xiaomi Mi 8 (red), Samsung S8 (blue) and Huawei P10 (green). The circle represents the R95, i.e. the radius of circle containing the $95 \%$ of the solutions. 
Longer sampling times have been tested, but the number of both L1/E1c and L5/E5a observations is always low. This leads us to state that it is still not possible to obtain reliable solutions through the ionosphere-free linear combination of observations from smartphones using PPP or RTK positioning techniques. This consideration has been made in the PPP analysis, but is still valid when RTK is used.

Table 2: Summary of RTK and PPP performances in terms of East, North and Up components measured by mean, Standard Deviation (STD) and Root Mean Square Error (RMSE) for the Xiaomi Mi 8 in Single Frequency (SF) and Dual Frequency (DF) modes, Samsung S8 and Huawei P10

\begin{tabular}{|l|l|c|c|c|c|c|c|c|c|c|}
\hline \multicolumn{3}{|c}{ Specifications } & \multicolumn{3}{|c|}{ East (cm) } & \multicolumn{3}{c|}{ North (cm) } & \multicolumn{3}{c|}{ Up (cm) } \\
\cline { 3 - 11 } & Mean & STD & RMSE & Mean & STD & RMSE & Mean & STD & RMSE \\
\hline \multirow{4}{*}{ RTK } & Mi 8 SF & -24 & 28 & 37 & -7.3 & 14 & 16 & 9.9 & 34 & 35 \\
\cline { 2 - 12 } & S8 & -7.0 & 26 & 27 & 27 & 49 & 56 & 1.8 & 20 & 180 \\
\cline { 2 - 12 } & P10 & -9.2 & 14 & 17 & -23 & 33 & 41 & 31 & 51 & 59 \\
\cline { 2 - 11 } & Mi 8 DF & -24 & 4.4 & 24 & 10 & 3.9 & 11 & 18 & 8.7 & 20 \\
\hline \multirow{3}{*}{$\begin{array}{l}\text { SF- } \\
\text { PPP }\end{array}$} & Mi 8 & -39 & 20 & 43 & -71 & 13 & 72 & -83 & 44 & 94 \\
\cline { 2 - 11 } & S8 & -110 & 56 & 120 & -320 & 40 & 320 & -84 & 34 & 91 \\
\cline { 2 - 11 } & P10 & -120 & 25 & 120 & -140 & 59 & 150 & -390 & 42 & 390 \\
\hline
\end{tabular}

\section{CONCLUSION}

This paper has outlined a future service for high accuracy positioning of the mass-market GNSS receivers. Known as FLAMINGO, the service takes advantage of recent developments in the GNSS industry, such as the introduction of Android raw GNSS measurements and introduction of the first dual frequency GNSS chipset, the BCM47755.

FLAMINGO targets sub-50cm aimed at applications including mapping and GIS data collection, AR tools, mobile location-based games and people tracking. Based on the client and server side, FLAMINGO will use many GNSS assets to improve positioning, such as multi-constellation, multi-signal GNSS, PPP, RTK, hybridized, rapid correction products and specialized reference station infrastructure.

There are significant challenges to overcome when operating in the mass-market environment. Hardware configuration in smartphones is not ideal for accurate GNSS, where typically the antenna is shared for other applications. Smartphone manufacturers also limit user control, preventing a developer from interacting with battery optimization routines and satellite tracking preferences.

Despite these challenges, impressive results can already be achieved as shown from testing of three smartphones, the dual frequency Xiaomi Mi 8, and single frequency Samsung S8 and Huawei P10. Accuracies approaching FLAMINGO's target are observed which are a significant improvement on the Smartphones' internal positioning solutions. Xiaomi Mi 8 results also demonstrated improvements that are delivered by the dual frequency chipset, not just via linear combination processing but also through the improvements to the L1/E1 acquisition and tracking.

There is still significant work ahead for FLAMINGO and high accuracy positioning for mass-market devices. However, what is presented is a bright and prosperous future for accurate GNSS in everyday life.

\section{ACKNOWLEDGMENTS}

This project has received funding from the European GNSS Agency under the European Union's Horizon 2020 research and innovation programme under grant agreement No 776436. The authors wish to thank the European Global Navigation Satellite Systems Agency for their support. 


\section{REFERENCES}

1. Malkos, S., "Google to provide raw GNSS measurements," GPS World, Summer 2016.

2. Cozzens, T., "Broadcom launches dual-frequency GNSS receiver for mass market," GPS World, Autumn 2017.

3. "World's first dual-frequency GNSS smartphone hits the market," European GNSS Agency, Summer 2018.

4. Trimble, “Trimble Catalyst Soft GNSS Solution,” Datasheet, 2017.

5. EOS Positioning Systems, “ARROW Gold for $1 \mathrm{~cm}$ RTK Accuracy,” Datasheet, Autumn 2017.

6. "Most popular Google Play categories," AppBrain, Winter 2018, [Online] Available: https://www.appbrain.com/stats/android-marketapp-categories, [Accessed: February 2018].

7. “GNSS Market Report Issue 5,” GSA, Spring 2017.

8. “Digital Market Report 2017 - Video Games,” Statista, Summer 2017.

9. “Google Play Store,” 42 Matters, Winter 2018, [Online] Available: https://42matters.com/stats, [Accessed: February 2018].

10. “2017 Connected Car,” Statista, Summer 2017.

11. “Automotive revolution - perspective towards 2030," McKinsey \& Company, Winter 2016.

12. Litman, T. A., "Automonous Vehicle Implementation Predictions - Implications for Transport Planning," Victoria Tranport Policy Unit, Summer 2018.

13. Pesyna, K. M., Heath, R. W., and Humphreys, T. E., "Centimeter positioning with a smartphone quality GNSS antenna," Radionavigation Laboratory Conference Proceedings, Tampa, FL, September 2014.

14. Siddakatte, R., Broumandan, A., and Lachapelle, G., "Performance evaluation of smartphone GNSS measurements with different antenna configurations," Presented at Royal Institute of Navigation Intenational Navigation Conference, Brighton, United Kingdom, November 2017.

15. Humphreys, T. E., Murrian, M. Pesyna, K. M., Podshivalov, S., and van Diggelen, F., "On the feasibility of cm-accurate positioning via a smartphone's antenna and GNSS chip," Radionavigation Laboratory Conference Proceedings, Savannah, GA, April 2016.

16. Realini, E., Caldera, S., Pertusini, L., and Sampietro, D., "Precise GNSS positioning using smart devices," Sensors, Vol. 17, No. 10, Autumn 2017, pp. 2434-2448.

17. Banville, S., and van Digglen, F., "Innovation: Precise positioning using raw GPS measurements from Android smartphones," GPS World, Autumn 2016.

18. Pirozzi, G., Mazzoni, A., Biagi, L., and Crespi, M., "Preliminary performance analysis with a GPS plus Galileo enabled chipset embedded in a smartphone," Proceedings of $30^{\text {th }}$ International Technical Meeting of the Satellite Division of The Institute of Navigation (ION GNSS+ 2017), Portland, OR, September 2017, pp. 101-115. 